Journal of the Operations Research

Society of Japan

Vol. 43, No. 3, September 2000

\title{
EFFICIENCY-MEASURING DEA MODEL FOR PRODUCTION SYSTEM WITH $k$ INDEPENDENT SUBSYSTEMS
}

\author{
Yinsheng Yang \\ Jilin University of Technology
}

\author{
Benjiang Ma \\ East China Jiaotong University
}

\author{
Masayuki Koike \\ University of Tsukuba
}

(Received March 11, 1998; Final April 10, 2000)

\begin{abstract}
Data Envelopment Analysis (DEA) is a mathematical programming approach to assess relative efficiencies with a group of decision making units (DMUs) such as production systems. There have been some useful models for their successful applications in many fields. In this paper, we first point out the defect of the first DEA model CCR (Charnes, Cooper and Rhodes, 1978) in measuring the efficiencies of the production system with $k$ independent subsystems and propose a new model YMK (Yang, Ma and Koike) by improving CCR model. Some properties and the relationship between CCR and YMK models are also discussed. It is concluded that the overall efficiency (YMK) of each DMU has a great deal to do with the efficiencies of its subsystems under CCR model. In fact, the overall efficiency value (YMK) of each DMU is equal to the maximum among the efficiency values of all its subsystems under CCR model. The examples given demonstrate the effectiveness of YMK model in measuring efficiencies of the production system with $k$ independent subsystems.
\end{abstract}

\section{Introduction}

Data Envelopment Analysis (DEA) was first introduced by Charnes and Cooper et al., famous operational researchers in U.S.A. Since the birth of the first model CCR in 1978 [2], other DEA models such as CCGSS [1], CCW [3], CCWH [4], GDEA [7] and uncertain models [5,6,8] have been established in succession. And with the development of its theory and application in many fields, DEA method has been proved being effective in evaluating and decision-making, especially in the efficiency-measurement of production systems with multi-input and multi-output.

However, there still exist various shortcomings in previous DEA models not only in theory but also in practice. For example, we have found that CCR model is not perfect even invalid in measuring the efficiency of the production system with $k$ independent subsystems. The following example will illustrate this aspect.

We first give the definition of the production system with $k$ independent subsystems (Figure 1). This kind of production system consists of $k$ independent production subsystems or $k$ independent production lines, and all inputs and outputs of $k$ subsystems constitute the overall input and output index system of the overall production system. For convenience, a production system with $k$ independent subsystems is to be abbreviated as $k$-ISPS. The same type of $n k$-ISPS refers to as a group of $n$ Decision-Making Units (DMUs) which has $k$ independent subsystems, and the numbers of inputs and outputs for every corresponding subsystem of $n k$-ISPS are identical.

By considering four 2-ISPS and each subsystem is single input and output DMU with the following input and output data (Figure 2). Taking DMU1 as an example, the input and output vectors for subsystems 1 and 2 become $(1,1)^{\mathrm{T}}$ and $(3,2)^{\mathrm{T}}$, respectively, and remaining subsystems can be evaluated similarly. 


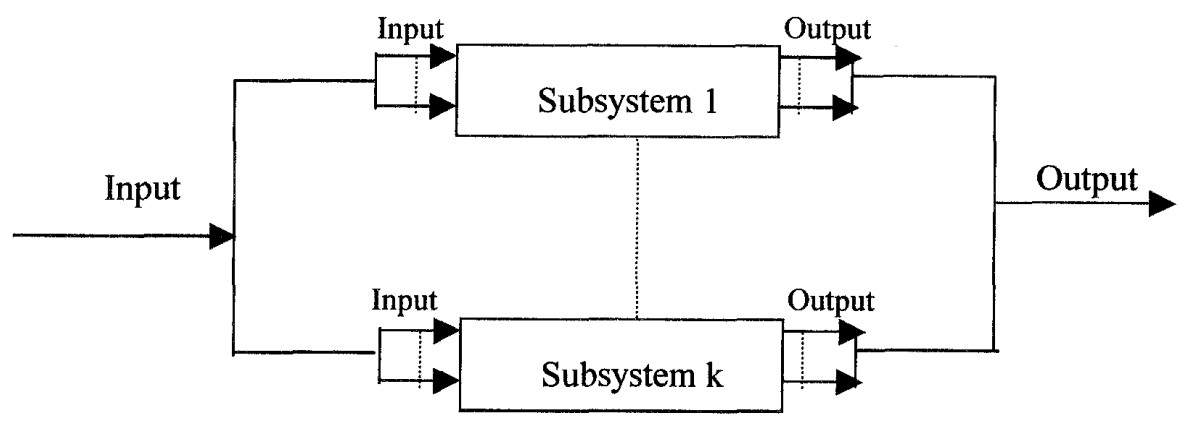

Figure 1 A production system with $k$ independent subsystems

Let us apply CCR model to evaluate their efficiencies under the following three cases:

(A) Subsystem 1 alone with four DMUs

(B) Subsystem 2 alone with four DMUs

(C) The overall system with four 2-ISPS

and their efficiency values are illustrated in Table 1.

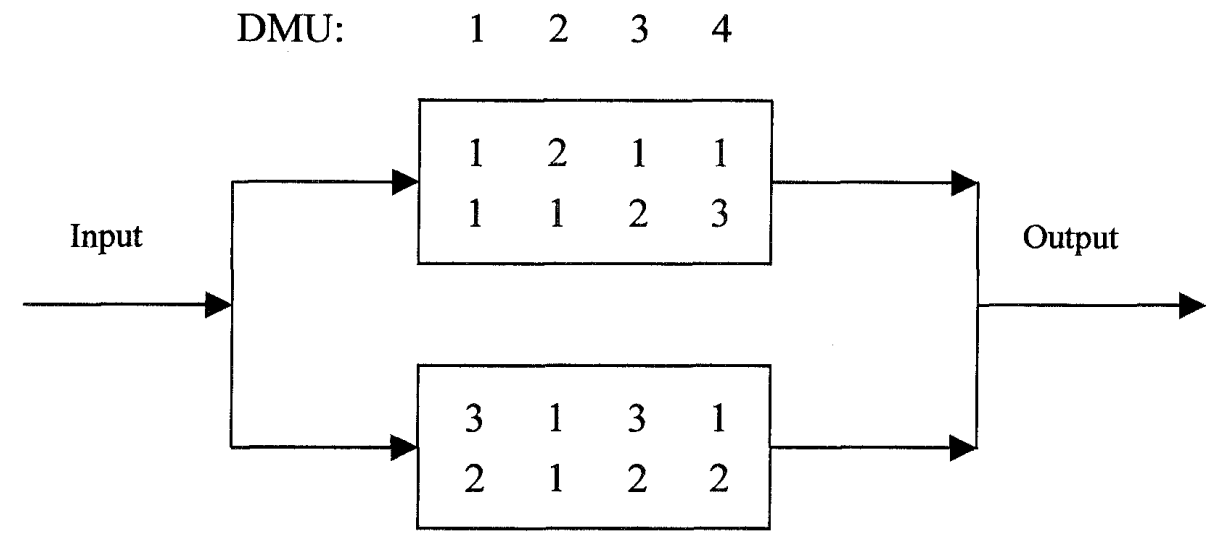

Figure 2 An example with four 2-ISPS

Table 1 Evaluating results for the example

\begin{tabular}{ccccc}
\hline Case number & DMU1 & DMU2 & DMU3 & DMU4 \\
\hline A & 0.333 & 0.167 & 0.667 & 1 \\
B & 0.333 & 0.5 & 0.333 & 1 \\
C & 1 & 0.5 & 1 & 1 \\
\hline
\end{tabular}

From Table 1, we can see that although DMU1 and DMU3 are efficient for the case number C, both case numbers A and B showed inefficient performance. The result illustrates that the overall efficient production system can also be improved in technical or scalar efficiency with the aid of information derived from other DMUs, which is just the shortcoming of CCR model in efficiency-measuring of $k$-ISPS. The purpose of the paper is to establish a new DEA model YMK for efficiency-measuring of $k$-ISPS by improving CCR model. So me properties and the relationship between CCR and YMK model will be discussed succeedingly in relation to theoretical and numerical example. 


\section{Efficiency - measuring DEA Model YMK for $\boldsymbol{k}$-ISPS}

Consider $n k$-ISPS and suppose the input and output vectors of the $i$ th subsystem belonging to the $j$ th DMU are $X_{j}^{(i)}, Y_{j}^{(i)}(\mathrm{i}=1, \ldots, \mathrm{k} ; \mathrm{j}=1, \ldots, \mathrm{n})$, respectively, and where $X_{j}^{(i)} \in E_{m_{i}}^{+}, Y_{j}^{(i)} \in E_{s_{i}}^{+}$, i.e. the numbers of the inputs and outputs for the $i$ th subsystem are $m_{i}$ and $s_{i}$, respectively. Let $m=\sum_{i=1}^{k} m_{i}, s=\sum_{i=1}^{k} s_{i}$, and $X_{j}^{T}=\left(X_{j}^{(1) T}, \ldots, X_{j}^{(k) T}\right) \in E_{m}^{+}$, $Y_{j}^{T}=\left(Y_{j}^{(1) T}, \ldots, Y_{j}^{(k) T}\right) \in E_{s}^{+}$are the overall input vector and output vector of the $j$ th DMU, respectively.

Obviously, since the overall production information is distributed into $k$ independent subsystems, we introduce the following definition.

Definition 1. Let $\overline{X_{i j}}=\left(0, \ldots, X_{j}^{(i) T}, \ldots, 0\right)^{T} \in E_{m}^{+}, \overline{Y_{i j}}=\left(0, \ldots, Y_{j}^{(i) T}, \ldots, 0\right) \in E_{s}^{+}$, i.e. the $i$ th component vector are the same with $X_{j}$ and $Y_{j}$, other component vectors are zero-vectors. The combination of $\left(\overline{X_{i j}}, \overline{Y_{i j}}\right)$ is referred to as the production gene of the $j$ th DMU.

Now let us consider the following mathematical programming which is called YMK DEA model.

$$
\begin{array}{ll}
\max & \frac{u^{T} Y_{0}}{v^{T} X_{0}}=V_{1} \\
\text { s.t. } & v^{T} \overline{X_{i j}}-u^{T} \overline{Y_{i j}} \geq 0 \\
& u \geq 0, v \geq 0 \\
& i=1, \ldots, k ; j=1, \ldots, n
\end{array}
$$

and where $X_{0}, Y_{0}$ (all positive) are known input and output vectors of the $j_{0}$ th DMU and $\mathrm{v}=$ $\left(v_{1}, \ldots, v_{m}\right)^{T}, u=\left(u_{1}, \ldots, u_{s}\right)^{T}$ (all non-negative) are the variable weight vectors to be determined by the solution of this programming problem.

By using Charnes-Cooper transformation

$$
t=\frac{1}{v^{T} X_{0}}, \omega=t v, \mu=t u
$$

the programming (1) can be changed into the following programming:

$$
\begin{array}{ll}
\max & \mu^{T} Y_{0}=V_{2} \\
\text { s.t. } & \omega^{T} \overline{X_{i j}}-\mu^{T} \overline{Y_{i j}} \geq 0 \\
& \omega^{T} X^{0}=1 \\
& \omega \geq 0, \mu \geq 0 \\
& i=1, \ldots, k ; j=1, \ldots, n
\end{array}
$$

Theorem 1: Fractional programming (1) is equivalent of linear programming (2) in the following sense:

(i) If $v^{0}$ and $u^{0}$ are the optimal solution of programming (1), then $\omega^{0}=t^{0} v^{0}$ and $\mu^{0}=t^{0} u^{0}$ are the optimal solution of programming (2) and their optimal values are identical, where $t^{0}=\frac{1}{v^{0 T} X_{0}}$.

(ii) If $\omega^{0}$ and $\mu^{0}$ are the optimal solution of programming (2), then $\omega^{0}$ and $\mu^{0}$ are the optimal solution of programming (1) and hence programming (1) has the same optimal objective value as programming (2).

Proof: see appendix.

Now we give the dual programming of programming (2) as follows:

$$
\min \theta=V_{3}
$$




$$
\begin{aligned}
& \quad \sum_{i, j} \overline{X_{i j}} \lambda_{i j}+s^{-}=\theta X_{0} \\
& \sum_{i, j} \overline{Y_{i j}} \lambda_{i j}-s^{+}=Y_{0} \\
& \lambda_{i j} \geq 0, s^{+} \geq 0, s^{-} \geq 0
\end{aligned}
$$

For programmings (2) and (3), we are obtainable the following Theorem.

Theorem 2 Both programmings (2) and (3) have optimal solution as well as equal optimal value and $V_{2}=V_{3} \leq 1$.

Proof: see appendix.

Definition 2 DMU- $j_{0}$ is said to be weak DEA efficient (YMK) if there exists an optimal solution $\left(\omega^{0}, \mu^{0}\right)$ of programming (2) such that $V_{2}=\mu^{0 T} Y_{0}=1$.

Definition 3 DMU- $j_{0}$ is said to be DEA efficient (YMK) if there exists an optimal solution $\left(\omega^{0}, \mu^{0}\right)$ of programming (2) such that $V_{2}=\mu^{0 T} Y_{0}=1$ and $\omega^{0}>0, \mu^{0}>0$.

By applying the duality theory of linear programming, the following theorem is easy to prove.

Theorem 3 DMU- $j_{0}$ is weak DEA efficient (YMK) if and only if the optimal value $V_{3}$ of programming (3) satisfies the condition that $V_{3}=1$. And DMU- $j_{0}$ is DEA efficient (YMK) if and only if every optimal solution $\lambda^{0}=\left(\lambda_{1}^{0}, \ldots, \lambda_{n}^{0}\right)^{T}, s^{0-}, s^{0+}, \theta^{0}$ of programming (3) satisfies the condition that $s^{0-}=0, s^{0+}=0, \theta^{0}=1$.

\section{The Relationship between CCR and YMK Model}

Consider the following CCR model for the overall production system DMU- $j_{0}$,

$$
\begin{array}{ll}
\max & \frac{u^{T} Y_{0}}{v^{T} X_{0}}=V_{4} \\
\text { s.t } & \frac{u^{T} Y_{j}}{v^{T} X_{j}} \leq 1, j=1, \ldots, n \\
& u \geq 0, v \geq 0
\end{array}
$$

where $X_{j}>0, Y_{j}>0$ are the input and output vectors of the $j$ th DMU, we have

Lemma 1 Each feasible solution of programming (1) is also feasible for programming (4), and objective values are identical.

Proof: Suppose $\left(u^{\prime}, v^{\prime}\right)$ is an arbitrary feasible solution of programming (1), thus

$$
u^{\prime T} \overline{Y_{i j}} \leq v^{\prime T} \overline{X_{i j}}, i=1, \ldots, k ; j=1, \ldots, n
$$

and

$$
\sum_{i=1}^{k} u^{\prime T} \overline{Y_{i j}} \leq \sum_{i=1}^{k} v^{\prime T} \overline{X_{i j}}, j=1, \ldots, n
$$

Noticing the structure of $\overline{X_{i j}}$ and $\overline{Y_{i j}}$, we have

$$
\sum_{i=1}^{k} u^{\prime T} \overline{Y_{i j}}=u^{\prime T} Y_{j}>0, \sum_{i=1}^{k} v^{\prime T} \overline{X_{i j}}=v^{\prime T} X_{j}>0, j=1, \ldots, n
$$

And hence, Eq. (5) is equivalent to

$$
\frac{u^{T} Y_{j}}{v^{T} X_{j}} \leq 1
$$

i.e. $\left(u^{\prime}, v^{\prime}\right)$ is also feasible for programming (4). Obviously, two objective values are identical.

According to Lemma 1, the following theorem is to be derived with ease.

Theorem $4 V_{1} \leq V_{4}$. Thus, if DMU- $j_{0}$ is weak DEA efficient (YMK), then it is also weak DEA 
efficient (CCR); If DMU- $j_{0}$ is DEA efficient (YMK), then it is also DEA efficient (CCR).

Next we will discuss the relationship between the efficiency value under YMK and efficiency values of $k$ subsystems under CCR.

Consider CCR model for evaluating $i$ th subsystem of DMU- $j_{0}$,

$$
\begin{array}{ll}
\max & \frac{u_{i}^{T} Y_{0}^{(i)}}{v_{i}^{T} X_{0}^{(i)}}=V_{1-i} \\
\text { s.t } & \frac{u_{i}^{T} Y_{j}^{(i)}}{v_{i}^{T} X_{j}^{(i)}} \leq 1, j=1, \ldots, n \\
& u_{i} \geq 0, v_{i} \geq 0
\end{array}
$$

where $X_{j}^{(i)}, Y_{j}^{(i)}$ are the input and output vectors of the $i$ th subsystem of DMU-j and $u_{i}, v_{i}$ denote corresponding output and input weight vectors of the $i$ th subsystem, respectively.

Lemma 2 If $p_{i}$ and $q_{i}$ are non-negative rational numbers such that

$$
\begin{aligned}
& \text { (i) } q_{i} \geq p_{i}, i=1, \ldots, k \\
& \text { (ii) } \sum_{i=1}^{k} q_{i}>0 ; \sum_{i=1}^{k} p_{i}>0
\end{aligned}
$$

let $I=\left\{i \mid i=1, \ldots, k\right.$ and $\left.q_{i} \neq 0\right\}$ and

then $\quad 0<\frac{\sum_{i=1}^{k} p_{i}}{\sum_{i=1}^{k} q_{i}} \leq \frac{p_{0}}{q_{0}} \leq 1$

$$
\frac{p_{0}}{q_{0}}=\max _{i \in I}\left\{\frac{p_{i}}{q_{i}}\right\}
$$

Lemma 3 If $p_{i}, q_{i}$ are positive rational numbers such that

$$
\begin{gathered}
\text { (i) } 0<\frac{p_{i}}{q_{i}} \leq 1, i=1, \ldots, k \\
\text { (ii) } \frac{\sum_{i=1}^{k} p_{i}}{\sum_{i=1}^{k} q_{i}}=1
\end{gathered}
$$

then $\frac{p_{i}}{q_{i}}=1(i=1, \ldots, k)$

Lemma 2 and Lemma 3 are to be proved with ease.

Theorem $5 \quad V_{1}=\max _{1 \leq i \leq k}\left\{V_{1-i}\right\}$

Proof: see appendix.

Theorem 6 ( $i$ ) DMU- $j_{0}$ is weak DEA efficient (YMK) if and only if there exists at least one in $k$ subsystems of DMU- $j_{0}$ which is weak DEA efficient (CCR) relative to the corresponding subsystems of other DMUs.

(ii) $D M U-j_{0}$ is DEA efficient (YMK) if and only if each subsystems of DMU- $j_{0}$ is DEA efficient (CCR) relative to the corresponding subsystems of other DMUs.

Proof: see appendix. 


\section{Examples}

Now we apply YMK model to the example shown in Figure 2 and provide the efficiency values of four 2-ISPS as follows (Table 2).

Table 2 Efficiency values (YMK) of the example

\begin{tabular}{lcccc}
\hline \multicolumn{1}{c}{ DMU } & 1 & 2 & 3 & 4 \\
\hline $\begin{array}{l}\text { The overall } \\
\text { efficiency } \\
\text { value(YMK) }\end{array}$ & 0.33 & 0.5 & 0.667 & 1 \\
\hline
\end{tabular}

As a practical example, the measurement of the overall efficiency of the macro-agricultural production system of China by using YMK model has been investigated. In fact, the macro-agricultural production system of China is practically considered to be the large-scale production system consisting of five subsystems such as cultivation, forestry, animal husbandry, fishery and rural enterprise which can be dealt with independent sector respectively (Figure 3). Obviously, all macro-agricultural production systems of thirty provinces, metropolises and autonomous regions in China constitute a typical thirty 5-ISPS. Therefore, we can apply YMK model to measure and compare the overall efficiencies of the macro-agriculture systems among thirty DMUs based on the statistical data.

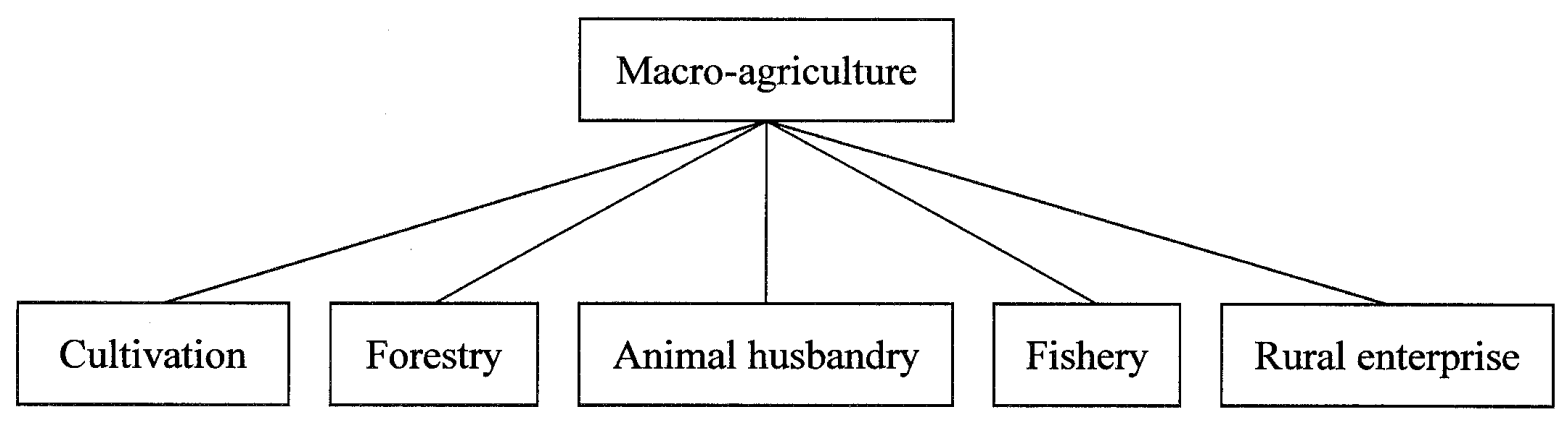

Figure 3 The structure of macro-agriculture

The following indicators can be selected as the input and output elements for each subsystem.

(1) Cultivation

Input: a) required labor force in cultivation (million person); b) arable area (million ha); c) material consumption cost in cultivation (million rmb, where rmb is the Chinese monetary unit).

Output : gross output in cultivation (rmb).

(2) Forestry

Input: a) required labor force in forestry (million person); b) forest area (million ha); c) material consumption cost in forestry ( $(\mathrm{mb})$.

Output : gross output in forestry (rmb).

(3) Animal husbandry

Input: a) required labor force in animal husbandry (million person); b) the number of maternal animals which have breeding capability; c) material consumption cost in animal husbandry (million rmb).

Output : gross output in animal husbandry (million rmb).

(4) Fishery

Input: a) required labor force in fishery (million person); b) fish-cultivating area (million ha); c) 


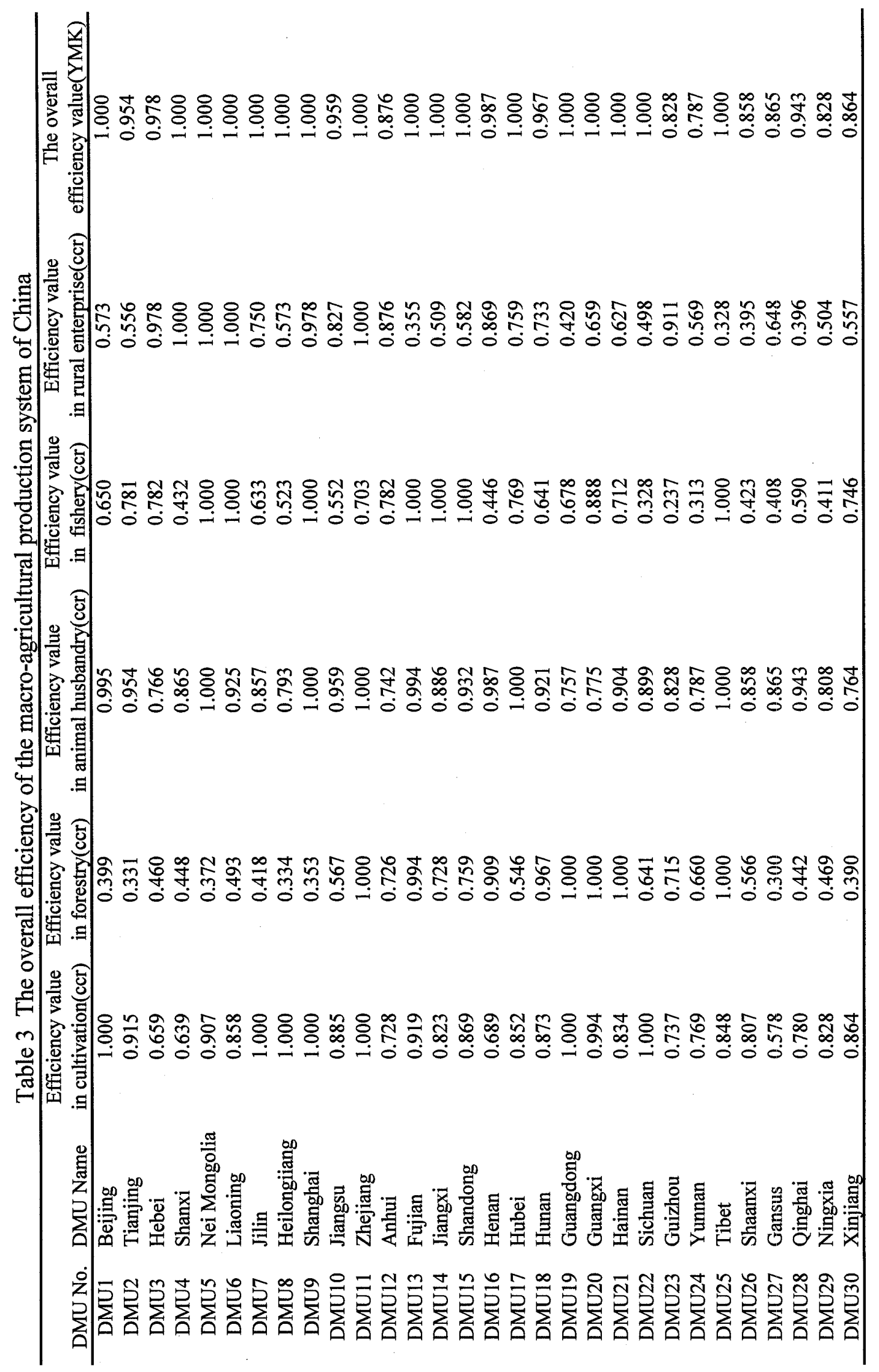


material consumption cost in fishery (million $\mathrm{rmb}$ ).

Output : gross output in fishery (million $\mathrm{rmb}$ ).

(5) Rural enterprise

Input: a) required labor force in rural enterprise (million person); b) the original value of fixed assets (million $\mathrm{rmb}$ ); $\mathrm{c}$ )working fund (million $\mathrm{rmb}$ ).

Output : profit and taxes (in unit of million $\mathrm{rmb}$ ).

Based on the input/output data coming from China Rural Statistical Yearbook 1997, we applied YMK model to evaluated the overall efficiencies of the macro-agriculture systems among thirty DMUs. The results are shown in Table 3. By analyzing these computational results, we can evaluate the overall performances of thirty DMUs. For example, we can not only rank thirty DMUs in overall performance, but also interpret their sector-developing harmony and equilibrium. Although the overall efficiency value of Liaoning province is equal to 1.000 , its efficiency value in forestry is only 0.493 , which shows that its overall development is no $t$ harmonious with five sectors.

\section{Conclusions}

From Table 1, Table 2 and Table 3, we can further verify the truth of Theorems described above. It is concluded that the overall efficiency (YMK) of each DMU has a great deal to do with the efficiencies of its subsystems under CCR model. In fact, Theorem 5 indicates that the overall efficiency value (YMK) of each DMU is equal to the maximum among the efficiency values (CCR) of all its subsystems. Comparing CCR and YMK models, YMK is more exact to be used in distinguishing efficient DMUs. Moreover, we also find that the inverse of Theorem 4 is not true by comparing Table 1 and Table 2, and thus the efficiency under YMK model is stronger than CCR model. We think that YMK model will be very effective for evaluating the overall efficiency of production systems with many subsystems, especially for complicated large-scale production systems such as agriculture systems.

Since Charnes, Cooper and Rhodes (1978) proposed the DEA approach for evaluation of relative efficiency, it has received considerable attention from both researchers and practitioners. One reason is that this approach has an advantage of evaluating DMUs under the most favorable conditions. It is not only for use as a tool for evaluation of past accomplishments but also as a tool to aid in planning future management. However, because of the existence of various circumstances in practice, the existing DEA models are restricted to some extent to be used in many cases. Therefore, further work on DEA will be of necessity from specific standpoints of both theoretical and practical approaches.

\section{Appendix}

\section{The proof of Theorem 1}

(i) For each feasible solution, $\omega \geq 0$ and $\mu \geq 0$, of programming (2) and the optimal solution, $v^{0}$ and $u^{0}$, of (1)

$$
\frac{u^{0 T} Y_{0}}{v^{0 T} X_{0}} \geq \frac{\mu^{T} Y_{0}}{\omega^{T} X_{0}}=\mu^{T} Y_{0}\left(\omega^{T} X^{0}=1\right)
$$

and

$$
\begin{aligned}
& \frac{u^{0 T} Y_{0}}{v^{0 T} X_{0}}=\mu^{0 T} Y_{0} \geq \mu^{T} Y_{0} \\
& \omega^{0}=t^{0} v^{0}=\frac{v^{0}}{v^{0 T} X_{0}}, \mu^{0}=t^{0} u^{0}=\frac{u^{0}}{v^{0 T} X_{0}}
\end{aligned}
$$

$\omega^{0}$ and $\mu^{0}$ are the feasible solution of programming (2) and thus also the optimal solution. 
Programmings (1) and (2) bring about identical optimal values.

(ii) If $\omega^{0}$ and $\mu^{0}$ are the optimal solution of programming (2), it is easy to know that $\omega^{0}$ and $\mu^{0}$ are to be the feasible solution of programming (1). For each arbitrary feasible solution $v, u$ of programming (1)

$$
\omega=t v, \mu=t u\left(t=\frac{1}{v^{T} X_{0}}\right)
$$

are the feasible solution of programming (2), thus we have

$$
\begin{gathered}
\mu^{0 T} Y_{0} \geq \mu^{T} Y_{0}=\frac{u^{T} Y_{0}}{v^{T} X_{0}} \\
\frac{\mu^{0 T} Y_{0}}{\omega^{0 T} X_{0}}=\mu^{0 T} Y_{0} \\
\frac{\mu^{0 T} Y_{0}}{\omega^{0 T} X_{0}} \geq \frac{u^{T} Y_{0}}{v^{T} X_{0}}
\end{gathered}
$$

therefore, $\omega^{0}$ and $\mu^{0}$ are to be the optimal solution of programming (1). (1) and (2) have the same optimal value.

\section{The proof of Theorem 2}

For (2), let

where

$$
\omega^{*}=\frac{X_{0}}{\left\|X_{0}\right\|^{2}}>0, \mu^{*}=\left(\mu_{1}^{*}, 0, \ldots, 0\right)^{T}
$$

$$
\mu_{1}^{*}=\min _{1 \leq j \leq n} \frac{\omega^{* T} \overline{X_{1 j}}}{\overline{y_{1 j}^{(1)}}}>0
$$

and $\left(\overline{X_{1 j}}, \overline{Y_{1 j}}\right)$ is the production gene of the 1st DMU as while $\overline{y_{1 j}^{(1)}}$ is the first component of $\overline{Y_{1 j}}$.

Obviously, $\omega^{*} \geq 0, \mu^{*} \geq 0$ and $\omega^{* T} X_{0}=1$. Noticing that only the first component of $\mu^{*}$ is not equal to zero,

$$
\omega^{{ }^{*} T} \overline{X_{i j}}-\mu^{* T} \overline{Y_{i j}}=\left\{\begin{array}{c}
\omega^{* T} \overline{X_{1 j}}-\mu_{1}^{*} \overline{y_{1 j}^{(1)}} \geq 0, i=1 \\
\omega^{* T} \overline{X_{i j}}>0, i=2, \ldots, k
\end{array}\right.
$$

therefore, $\omega^{*}$ and $\mu^{*}$ are the feasible solution of programming (2).

For programming (3), let

$$
\begin{aligned}
\lambda_{i j} & =\left\{\begin{array}{l}
1, j=j_{0} \\
0, j \neq j_{0}
\end{array}\right. \\
s^{+} & =0, s^{-}=0, \theta=1
\end{aligned} \quad i=1, \ldots, k ; j=1, \ldots, n
$$

then they are the feasible solution of programming (3). According to the duality theory, both programmings (2) and (3) have optimal solution and exhibit equal optimal value.

And from $\omega^{T} \overline{X_{i j_{0}}}-\mu^{T} \overline{Y_{i j_{0}}} \geq 0, i=1, \ldots, k$, we have

$$
\sum_{i=1}^{k}\left(\omega^{T} \overline{X_{i j_{0}}}-\mu^{T} \overline{Y_{i j_{0}}}\right)=\omega^{T} X_{0}-\mu^{T} Y_{0} \geq 0
$$

i.e.

$$
\mu^{T} Y_{0} \leq \omega^{T} X_{0}=1
$$

thus

$$
V_{3}=V_{2} \leq 1
$$




\section{The proof of Theorem 5}

Suppose $\left(u^{\prime}, v^{\prime}\right)$ is an arbitrary feasible solution of programming (1) and assemble it according to $k$ subsystems as follows:

$$
\begin{aligned}
& u^{\prime}=\left(u_{1}^{\prime T}, \ldots, u_{k}^{T}\right)^{T} \\
& v^{\prime}=\left(v_{1}^{\prime T}, \ldots, v_{k}^{T}\right)^{T}
\end{aligned}
$$

From the structure of $\left(\overline{X_{i j}}, \overline{Y_{i j}}\right)$ and that

$$
v^{\prime T} \overline{X_{i j}}-u^{\prime T} \overline{Y_{i j}} \geq 0
$$

we have

$$
v_{i}^{T} X_{j}^{(i)}-u_{i}^{T} Y_{j}^{(i)} \geq 0, i=1, \ldots, k ; j=1, \ldots, n
$$

and

$$
\begin{gathered}
v^{\prime T} X_{j_{0}}=\sum_{i=1}^{k} v_{i}^{T} X_{j_{0}}^{(i)}>0 \\
u^{\prime T} Y_{j_{0}}=\sum_{i=1}^{k} u_{i}^{\prime T} Y_{j_{0}}^{(i)}>0
\end{gathered}
$$

let $\mathrm{I}=\left\{i \mid i=1, \ldots, k\right.$ and $\left.v_{i}^{T T} X_{j_{0}}^{(i)} \neq 0\right\}$ and

$$
\frac{u_{i_{0}} T Y_{j_{0}}^{\left(i_{0}\right)}}{v_{i_{0}}^{\prime T} X_{j_{0}}^{\left(i_{0}\right)}}=\max _{i \in I}\left\{\frac{u_{i}^{\prime T} Y_{j_{0}}^{(i)}}{v_{i}^{T} X_{j_{0}}^{(i)}}\right\}
$$

From Eq.(6) and Lemma 2, we note that $\left(u_{i_{0}}^{\prime}, v_{i_{0}}^{\prime}\right)$ is feasible for programming $\left(1-i_{0}\right)$ and

$$
0<\frac{u^{\prime T} Y_{j_{0}}}{v^{T} X_{j_{0}}} \leq \frac{u_{i_{0}}^{\prime T} Y_{j_{0}}^{\left(i_{0}\right)}}{v_{i_{0}}^{T} X_{j_{0}}^{\left(i_{0}\right)}} \leq 1
$$

i.e. $0<V_{1-i} \leq 1$.

Notice the arbitrariness of $u^{\prime}$ and $v^{\prime}$, we have

$$
0<V_{1} \leq V_{1-i_{0}} \leq \max _{1 \leq i \leq k}\left\{V_{1-i}\right\}
$$

With no loss of generality, suppose

$$
V_{1-1}=\max _{1 \leq i \leq k}\left\{V_{1-i}\right\}
$$

and $\left(u_{1}^{0}, v_{1}^{0}\right)$ is the optimal solution of programming $(1-1)$. Let $u^{0}=\left(u_{1}^{0 T}, 0, \ldots, 0\right)^{T}$, $v^{0}=\left(v_{1}^{0 T}, 0, \ldots, 0\right)^{T}$, then $\left(u^{0}, v^{0}\right)$ is a feasible solution of programming (1) and the objective function value is

$$
\frac{u^{0 T} Y_{j_{0}}}{v^{0 T} X_{j_{0}}}=\frac{u_{1}^{0 T} Y_{j_{0}}^{(1)}}{v_{1}^{0 T} X_{j_{0}}^{(1)}}=V_{1-1}
$$

From $V_{1} \leq V_{1-1}$, we have $V_{1}=V_{1-1}=\max _{1 \leq i \leq k}\left\{V_{1-i}\right\}$.

\section{The proof of Theorem 6}

From Theorem 5, (i) is obvious and the sufficiency of (ii) is also very easy to prove. Now we only prove the necessity of (ii).

Because DMU- $j_{0}$ is DEA efficient (YMK), there exists an optimal solution $u^{0}>0, v^{0}>0$ of (1) such that

$$
\begin{aligned}
& \frac{u^{0 T} Y_{j_{0}}}{v^{0 T} X_{j_{0}}}=1 \\
& v^{0 T} \overline{X_{i j}}-u^{0 T} \overline{Y_{i j}} \geq 0, i=1, \ldots, k ; j=1, \ldots, n .
\end{aligned}
$$

Now block $u^{0}$ and $v^{0}$ according to $k$ subsystems and let 


$$
\begin{aligned}
& u^{0}=\left(u_{1}^{0 T}, \ldots, u_{k}^{0 T}\right)^{T} \\
& v^{0}=\left(v_{1}^{0 T}, \ldots, v_{k}^{0 T}\right)^{T}
\end{aligned}
$$

then $u_{i}^{0}>0, v_{i}^{0}>0, i=1, \ldots, k$.

Notice the structure of $\overline{X_{i j}}, \overline{Y_{i j}}$ and from Eq.(7) and (8) we have

$$
\begin{array}{ll} 
& v_{i}^{0 T} X_{j}^{(i)}-u_{i}^{0 T} Y_{j}^{(i)} \geq 0 \\
\text { i.e. } \quad & 0<\frac{u_{i}^{0 T} Y_{j}^{(i)}}{v_{i}^{0 T} X_{j}^{(i)}} \leq 1, i=1, \ldots, k ; j=1, \ldots, n \\
\text { and } \quad & \frac{u^{0 T} Y_{j_{0}}}{v^{0 T} X_{j_{0}}}=\frac{\sum_{i=1}^{k} u_{i}^{0 T} Y_{j_{0}}^{(i)}}{\sum_{i=1}^{k} v_{i}^{0 T} X_{j_{0}}^{(i)}}=1
\end{array}
$$

and

According to Lemma 3, and Eq.(9), (10), $\left(u_{i}^{0}, v_{i}^{0}\right)$ is a feasible solution of (1-i) such that

$$
V_{1-i}=\frac{u_{i}^{0 T} Y_{j_{0}}^{(i)}}{v_{i}^{0 T} X_{j_{0}}^{(i)}}=1
$$

Therefore the $i$ th subsystem is DEA efficient (CCR).

\section{Acknowledgments}

The authors wish to thank the referees and the Editor-in-Chief for their perceptive comments and recommendations.

\section{References}

[1] A.Charnes, W.W.Cooper, B.Golany, L.Seiford and J.Stutz: Foundation of data envelopment analysis for Pareto-Koopmans efficient empirical production. Journal of Econometrics (Netherlands), 30 (1985) 91-107.

[2] A.Charnes, W.W.Cooper and E.Rhodes: Measuring the efficiency of decision making units. European Journal of Operational Research, 12 (1978) 429-444.

[3] A.Charnes, W.W.Cooper and Q.L.Wei: A Semi-infinite multicriteria programming approach to data envelopment analysis with infinitely many decision-making units. The University of Texas at Austin, Center for Cybernetic Studies, Report CCS 551 (September, 1986).

[4] A.Charnes, W.W.Cooper, Q.L.Wei and Z.M.Huang: Cone ratio data envelopment analysis and multi-objective programming. International Journal of Systems Science, 20 (1989) 1099-1118.

[5] J.K.Sengupta: Data envelopment analysis for efficiency measurement in the stochastic case. Computers and Operations Research, 14 (1987) 117-129.

[6] Y.S.Yang, L.Li and H.L.Gao: DEA model for grey systems and its application. Proceedings Intern. AMSE Conference "Modeling, Simulation \& Control" (USTC Press, Hefei, China, 1993), 1577-1587.

[7] G.Yu, Q.L.Wei and P.Brockett: A generalized data envelopment analysis model: A unification and extension of existing methods for efficiency analysis of decision-making units. Annals of Operation Research, 66 (1996) 47-92.

[8] K.Y.Zhang, Y.S.Yang and L.H.Li: Data envelopment analysis model with fuzzy numbers. Proceedings of The Third China-Japan International Symposium on Industrial Management (International Academic Publishers, Beijing, China, 1996), 431-436. 


\section{Yinsheng Yang}

Department of Applied Mathematics

Jilin University of Technology

Changchun 130025, P.R.China

E-mail: yys0729@public.cc.jl.cn 